\title{
Test-taking Processes of Vocabulary Tests in Context From the Perspective of Think-Aloud Analysis
}

\author{
Yuko Morimoto \\ Graduate School, University of Tsukuba \\ The Japan Society for the Promotion of Science
}

\section{Introduction}

Vocabulary tests in context, rather than just presenting target words in isolation, are now popular methods to assess knowledge of word meanings. Although some objections have been suggested against vocabulary tests in context (e.g., Laufer, Elder, Hill \& Congdon, 2004), there is a strong current toward contextualized tests because of their authenticity and reliability (Hughes, 2003). However, there is still much room for research about vocabulary tests in context as we can see from Read's (2000) words: "despite the lack of research evidence on the role of context in vocabulary assessment, it has become almost an article of faith among both language teachers and testers that vocabulary should always be presented in context" (p. 101). Some of the untouched areas include the constructs of these tests, factors affecting item difficulty, and students' test-taking processes. Of these areas, this study paid special attention to the latter two and investigated how test-takers used contexts to reach correct answers and how they chose-one particular answer from the choices, by comparing the test-taking processes for easy and difficult items. In this way, it was possible to elucidate what makes contextualized vocabulary tests more difficult from the perspective of test-takers' actions and thoughts during the tests.

Some studies treated vocabulary tests in context from a viewpoint of speededness (Schedl, Thomas \& Way, 1995), construct definition (Morimoto, 2006a; Perkins \& Linnville, 1987; Pike, 1979), and difficulty (Henning, 1991; Mori, 2002; 2003; Morimoto, 2006b). Since this study focuses on the third perspective, research that covers issues on difficulty is reviewed. Henning (1991) used eight vocabulary tests differing in the length of context and test types to compare their difficulty, reliability, validity, and familiarity of students with the test formats. His study revealed that matching-formats had higher estimates of reliability and were significantly easier than supplying-formats, and also found that familiarity of test-formats did not have a significant influence on the results. On the other hand, Mori (2002, 2003) compared three kinds of tests for identifying novel word meaning: presenting a target word in isolation (target words only), presenting context and leaving the target word blank (contexts only), and presenting a target word in context (both target words and contexts). Her result suggested that presenting both target words and contexts led to a significantly better result than presenting either one of them alone, so both target words and contexts played additional roles as clues. This research was supported by Morimoto's (2006b) 
study which compared the difficulties of three kinds of multiple-choice vocabulary tests in context. These tests were ones in which: (a) only the target words could be clues; (b) only the contexts could be clues; and (c) both the target words and the context could be clues. Her research revealed that (a) and (b) had similar difficulty, and (c) was significantly easier than both (a) and (b). What this result indicated was that the contexts can be as effective clues as target words in the tests when either can be clues. However, because of their quantitative methods, neither study went any further in terms of how the contexts were used by test-takers and what kinds of context were more effective.

The information in the contexts largely affects the difficulty of identifying target words since research has revealed that encountering richer contexts provides a higher probability of inferring unknown words, predicting known words, and pronouncing target words correctly (Beck, McKeown, \& McCaslin, 1983; Juel, 1980; Mondria \& Boer, 1991; Pearson \& Studt, 1975). In an attempt to examine what kind of contextual information learners actually use, some researchers have made a framework for classifying the use of context through studying lexical inferencings (e.g., Ames, 1966; Bengeleil \& Paribakht, 2004; Haastrup, 1991; McCullough, 1958; Sternberg \& Powell, 1983). Lexical inferencing and vocabulary tests in context have the same feature that they both includes target words in context, and learners have to utilize information from contexts and the target words themselves. Of course, tests of lexical inferencing and vocabulary tests have different characteristics. For example, there is a higher probability that test-takers will know the target words in a vocabulary test if it is of matching-format than lexical inferencing. In order to make vocabulary tests in context more comparable with lexical inferencing, this study used the vocabulary test in context with the supplying format as shown below.

\section{Her charming ( ) has won her numerous admirers.}
a. character*
b. disease
c. peace
d. promise

This test has one blank instead of a target word in each context; hence, test-takers have to use contexts in order to reach correct answers, in a way similar to lexical inferencing. In this study, the focus is more on the use of contexts rather than target words, so the classifications of contextual use from lexical inferencing will be applicable in this study.

Of the frameworks presented above, this study used Haastrup's (1991) and Ames's (1966), since both of these made their classifications through the data from think-aloud protocols which would be used in this study. Other classifications (e.g., McCullough, 1958; Sternberg \& Powell, 1983) were made from teachers' intuition, and Bengeleil and Paribakht's (2004) framework has less interlingual sources than Haastrup's, so the data-based and more detailed frameworks better suited for classifying test-taker's use of clues. Haastrup divided the clues into three categories: contextual, 
intralingual, and interlingual clues, as shown in Table 1. Although this is finely classified framework and used by researchers of lexical inferencing, there is a different tendency of classification between contextual clues and other clues. Intralingual and Interlingual clues were classified by knowledge sources (i.e., types of clues), whereas contextual clues were divided based on where the clues were. Therefore, these three kinds of clues might not be perfectly comparable. Therefore, Ames's framework which focused on the knowledge sources of contextual clues was used to complement Haastrup's framework. This classification is presented in Table 2.

Table 1

Haastrup's (1991) Framework

Contextual The Text I-1 One or two words from the immediate co-text clues

I-2 The immediate co-text

I-3 A specific part of the co-text beyond the sentence of the test word

I-4 Unspecified use of the text

Knowledge of $\mathrm{I}-5$

the world

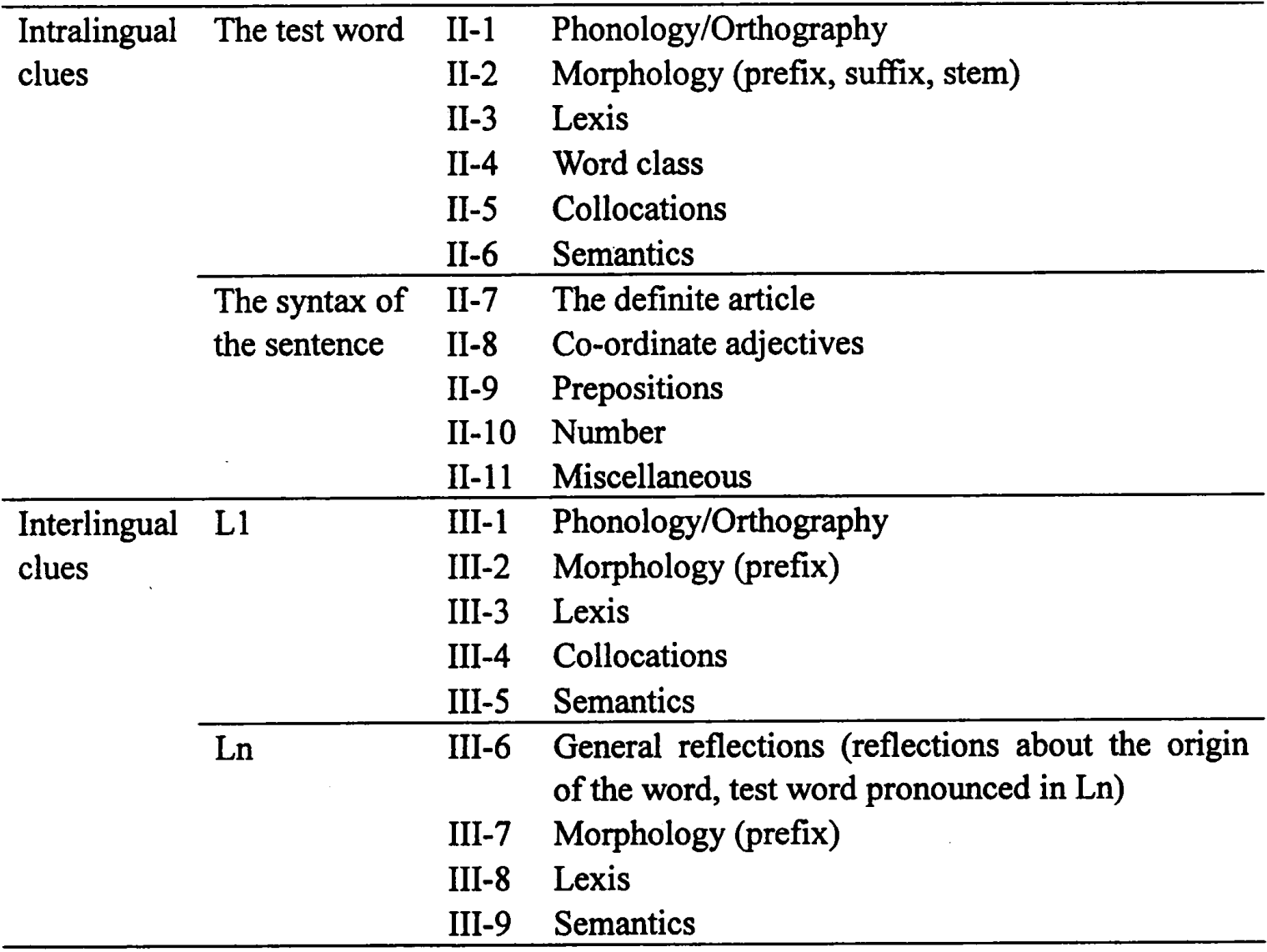

Note. Clues coded as contextual clues were further divided according to Ames (1966). 
Table 2

\begin{tabular}{ll} 
Ames's (1966) Framework \\
\hline I & Clues derived from language experience or familiar expressions \\
II & Clues utilizing modifying phrases or clauses \\
III & Clues utilizing definition or description \\
IV & Clues provided through words connected or in series \\
V & Comparison or contrast clues \\
VI & Synonym clues \\
VII & Clues provided by the tone, setting, and mood of a selection \\
VIII & Referral clues \\
IX & Association clues \\
X & Clues derived from the main idea and supporting details pattern of paragraph \\
& organization \\
XI & Clues provided through the question-and-answer pattern of paragraph \\
& organization \\
XII & Preposition clues \\
XIII & Clues utilizing non-restrictive clauses or appositive phrases \\
XIV & Clues derived from cause and effect pattern of paragraph and sentence \\
& organization
\end{tabular}

However, contexts are not the only factor affecting item difficulty in multiple-choice vocabulary tests in context. Another important factor is quality of choices (e.g., Goodrich, 1977; Greidanus \& Niehnhuis, 2001; Marshalek, 1981; Mori, 1999). Of these studies, Goodrich used contextualized vocabulary tests and showed that distractors which could fit the context were more plausible to his participants than any other distractors, such as antonyms or arbitrarily chosen words, so it could be inferred that contexts and distractors had a strong relationship. Mori prepared four choices in her research to investigate how her participants used the clue in the target words and in the contexts to infer novel word meaning. These choices were: (a) kanji distractor, which is consistent with the meanings of the component character of target words, but not consistent with the context; (b) context distractor, which is consistent with the context but not with the target words' component; (c) anomalous distractor, which is inconsistent with both the target words and the context; and (d) the correct answer. However, she used these distractors only for classifying her students by their learning styles. Therefore, the number of studies about vocabulary treating 'both contexts and distractors is limited, and few studies, if any, have investigated how test-takers select one choice in vocabulary tests.

Based upon the limitations of previous studies, the purpose of this study was to investigate what types of context clues test-takers used when they took vocabulary tests in context, and also to investigate how they made both their correct and incorrect choices by comparing the test-taking processes between easy and difficult items. In order to investigate these two issues, two research questions were set. 
RQ1: Is there any difference of use of context clues between easy and difficult items of vocabulary tests in context?

RQ2: Is there any difference in the processes of choosing an answer in multiple-choice vocabulary tests in context between easy and difficult items?

\section{Method}

\subsection{Preliminary study}

The preliminary study was conducted to select both easy and difficult items beforehand. A total of 66 first-year university students took the multiple-choice vocabulary tests in context. The tests consisted of 40 items, and had to be completed in 15 minutes. This test had a supplying-format and four choices for each item; therefore, participants had to read the context and select one correct answer which was the best of the four choices. All the items were made by the researcher and were checked by an English native speaker.

The mean score was 23.58 out of a maximum possible score of $40(S D=7.08)$, which means the proportion correct was $58.95 \%$. Of the 40 items, eight easy items and 10 difficult items were chosen for the main study. The descriptive statistics are presented in Table 3. There was a significant difference of scores between easy and difficult items $(t(16)=12.19, p=.000)$.

Table 3

Descriptive Statistics of the Items Selected for the Main Study

\begin{tabular}{lrccc}
\hline & $k$ & $\begin{array}{c}\text { Mean Proportions } \\
\text { Correct }\end{array}$ & $S D$ & Range \\
\hline Easy Items & 8 & $71.64 \%$ & 7.04 & $65.67-88.06 \%$ \\
Difficult Items & 10 & $38.21 \%$ & 3.87 & $32.84-44.78 \%$ \\
\hline
\end{tabular}

\subsection{Participants}

Twenty-one university students participated in the main study and none of them took part in the preliminary study. Their majors were diverse, including subjects such as English education, physical education, agrobiological resources, sociolinguistics, and nursing and medical technology. There were 11 first-year students, two sophomores, six juniors and four seniors. They were all paid-volunteers.

\subsection{Materials and Procedures}

Data was collected individually. The items were presented one by one in Microsoft PowerPoint 2003. Firstly, the researcher explained to the participants how and what would be investigated in the study and the participants then gave prior consent to their voices being recorded. Before participants started taking the test, the procedure of think-aloud was introduced to them by the researcher demonstrating, in which they 
role-played how to think-aloud by doing a little arithmetic. By doing this, each participant got used to how to verbalize their thought. After that, they took 18 vocabulary items, each of which had a pair of brackets in a sentential context, selected in the preliminary study. Limiting the length of contexts allowed us to answer more items in a limited time, so contexts longer than a sentence were not used. First, the participants were shown only the stem of the vocabulary items without choices in order to examine clearly how they used the contextual information. After that, the four choices (one correct answer and three distractors) appeared and the participants chose one when they could. They were asked to answer by thinking-aloud, and when they remained silent or did not state why they had selected one answer, the researcher intervened and asked some questions for confirmation. The participants were allowed to leave the item unanswered if they could not select one choice, but they were asked to delete distractors when they could.

\subsection{Data Analysis}

After writing down the recorded protocol, two graduate students who had majored in applied linguistics used Haastrup's (1991) and Ames's (1966) classification schemes to independently code one third of the used clues. Firstly, we classified the clues based on Haastrup's, except for I-3 and I-4 because these clues are for contexts beyond the sentence, and this study used sentential contexts, so no clues were categorized in either of I-3 and I-4 in this study. When any of the clues belonged to the contextual categories in Haastrup's, Ames's framework was used to classify in more detail. When there were ambiguous points, an English native speaker checked and resolved them. The agreement between the two raters was $89.88 \%$. Comparing this with Ames's inter-rater reliability (Cronbach's alpha $=.80$ ), the obtained degree of agreement was acceptable; hence, the remaining two thirds were classified by the researcher alone.

In conducting the analysis, the proportions correct, not the raw data, were used. This was because some participants had accidentally missed some items and had consequently been eliminated from the analysis for those items; therefore, the total number of test-taker for each item sometimes varied.

\section{Results and Discussion}

\subsection{Use of the Contextual Information}

Figure 1 describes the proportions of Haastrup's (1991) clues used by participants. Overall, in both items, there is the same tendency that they heavily depended on I-2 (immediate co-text), which supported the results of lexical inferencing in previous studies (e.g., Aizawa, 1998; Takayama, 2003). 


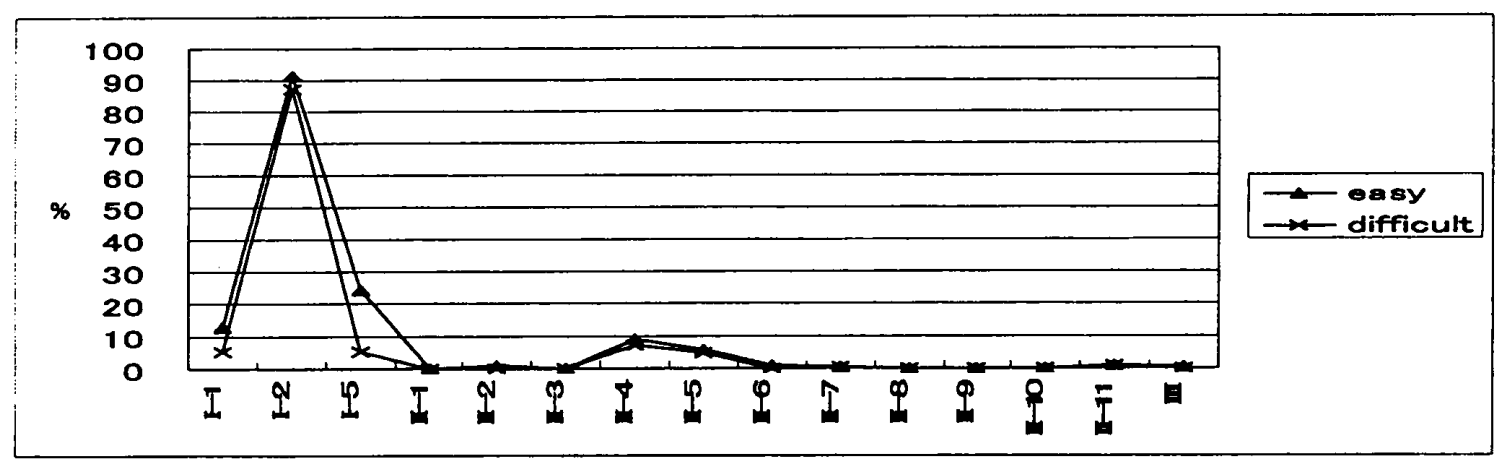

Figure 1. Haastrup's (1991) clues used by participants

Two-way ANOVAs (difficulty $\times$ types of clues) were conducted except for II-1, II-3, II-8, and II-9, which were not used at all. Furthermore, interlingual clues (III in Table1) were seldom used, so III was not subcategorized but was calculated as a whole. The result indicated that there was a significant main effect of difficulty $(F(1,16)=$ $\left.4.77, p=.044, \eta^{2}=.081^{1)}\right)$, and also the main effect of types of clues $(F(3.31,52.93)=$ $\left.160.95, p=.000, \eta^{2}=.991\right)$, but the interaction was not significant $(F(3.31,52.93)=$ $1.28, p=.290, \eta^{2}=.006$ ). This result means that the participants in this study used more clues when they answered easy items than when they answered difficult items, and they used I-2 (the immediate co-text) more frequently than all the other clues. The result of multiple comparisons indicates that II-4 (word class) was used more than some of the clues (II-6, II-9, II-10, and III).

Table 4

Descriptive Statistics of Used Clues Based on Haastrup's (1991) Framework

\begin{tabular}{|c|c|c|c|c|c|c|c|}
\hline & Difficulty & Mean & $S D$ & & Difficulty & Mean & $S D$ \\
\hline \multirow[t]{3}{*}{$\mathrm{I}-1$} & easy & 13.69 & 17.35 & II-6 & easy & 0.60 & 1.68 \\
\hline & difficult & 6.21 & 11.67 & & difficult & 0.00 & 0.00 \\
\hline & total & 9.54 & 14.51 & & total & 0.26 & 1.12 \\
\hline \multirow[t]{3}{*}{$\mathrm{I}-2$} & easy & 91.07 & 10.93 & II-7 & easy & 0.60 & 1.68 \\
\hline & difficult & 84.26 & 12.28 & & difficult & 0.50 & 1.58 \\
\hline & total & 87.29 & 11.88 & & total & 0.54 & 1.58 \\
\hline \multirow[t]{3}{*}{$\mathrm{I}-5$} & easy & 18.45 & 24.34 & II-10 & easy & 0.00 & 0.00 \\
\hline & difficult & 6.21 & 8.10 & & difficult & 0.48 & 1.51 \\
\hline & total & 11.65 & 17.83 & & total & 0.26 & 1.12 \\
\hline \multirow[t]{3}{*}{ II-2 } & easy & 1.19 & 2.20 & II-11 & easy & 1.19 & 2.20 \\
\hline & difficult & 0.00 & 0.00 & & difficult & 1.43 & 3.21 \\
\hline & total & 0.53 & 1.54 & & total & 1.32 & 2.74 \\
\hline \multirow[t]{3}{*}{ II-4 } & easy & 5.36 & 5.94 & III & easy & 0.60 & 1.68 \\
\hline & difficult & 8.62 & 6.67 & & difficult & 0.00 & 0.00 \\
\hline & total & 7.17 & 6.39 & & total & 0.26 & 1.12 \\
\hline \multirow[t]{3}{*}{ II-5 } & easy & 5.36 & 8.98 & & & & \\
\hline & difficult & 5.71 & 8.92 & & & $\cdot$ & \\
\hline & total & 5.56 & 8.68 & & & & \\
\hline
\end{tabular}


The above result is reasonable because if the participants could use more clues, these items were more likely to become easy. In order to see this effect more clearly, the number of clues used was examined, as illustrated in Figure 2.

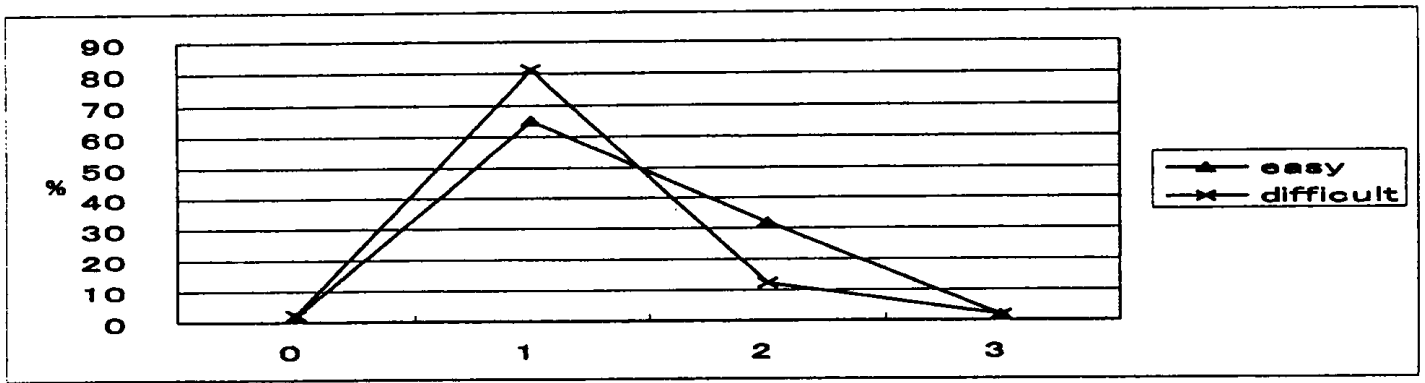

Figure 2. The number of clues used by participants in the vocabulary test

It is obvious that in easy items, more participants used plural, that is, two clues, whereas in difficult items they mostly used only one clue. Considering that the participants used I-2 about $90 \%$ of the time both in easy and difficult items (see Figure 1 above), it can be understood that the participants' first option was to use the immediate co-text (I-2), and there were other clues following. In most cases, their second option was I-5 because of the difference of about $20 \%$ shown in Figure 1. Below are examples of protocol data.

Your jeans will ( ) a little once you start wearing them.

a. debate b. expand* c. identify d. organize

$<$ participant \#4 (using I-2 and I-5) $>$ 2)

"WELL...JEANS...I CANNOT RECOGNIZE HOW THIS once, once IS USED, BUT ANYWAY them INDICATES JEANS, SO PERHAPS, WHEN STARTING wearing, START WEARING THE JEANS, WELL...LET ME SEE...little, a little...WHY DO THEY BECOME SMALL? DOES IT MEAN A LITTLE BIT OR SOMETHING LIKE THAT? WHEN START WEARING SOMETHING, I THINK IT WILL BECOME BIG. I DON'T KNOW."

$<$ participant \#7 (using I-2 and I-5)>

"WELL, THIS MEANS, WELL, ONCE START WEARING JEANS, little, THEY BECOMES DIRTY A LITTLE OR THEY SHRINK. NO, THEY SHRINK AFTER WASHING..."

Participant \#4 first relied on I-2 and reached an incorrect answer: shrink or become small, since she was confused by the phrase a little. She regarded a little as meaning 'becoming small', although it actually modifies the verb which should be in the brackets. However, after that, she changed her answer by using her world 
knowledge that jeans or clothes tend to stretch after we wear them. Another example is participant \#7, who also used both I-2 and I-5. Although she did not do so explicitly, this participant modified her own answer, shrink, by using her background knowledge because she thought that clothes shrink after being washed. To sum up from the protocol above, although using additional clues did not always make participants reach correct answers, the clues at least helped test-takers narrow down the possible answers. Therefore, it can be inferred that the participants in this study mainly used I-2, immediate co-text, and II-4, word class, but when available, they also used the clues of world knowledge complementally. Whether or not items are easy possibly depends on whether world knowledge can give clues to reach correct answers.

However, it should be noted that this is not only a determinant of easy items, since in more than $60 \%$ of cases, the participants used only one clue in answering easy items (see Figure 2), which indicates that the contexts themselves in easy items may have the characteristic of making the items easy. In an attempt to examine the effect of context, the context clues used by the participants were further divided by Ames's (1966) framework, which is illustrated in Figure 3. As with Haastrup's (1991) framework above, two-way ANOVAs were conducted for II, V, VII, IX, and XII since other clues were not used by any of the participants. The results showed that there was no significant interaction, $\left(F(2.62,41.67)=.70, p=.537 \eta^{2}=.002\right)$, but there were significant main effects of clue types $\left(F(2.62,41.67)=34.35, p=.000, \eta^{2}=.821\right)$ and difficulty $\left(F(1,16)=11.71, p=.003, \eta^{2}=.349\right)$. The result of multiple comparisons implied that VII (clues provided by the tone, setting, and mood of a selection) was significantly more used than any other types of contextual clues, and there was no other significant difference. Furthermore, clues were more frequently used in easy items than in difficult items, which was the same tendency observed when using Haastrup's classification.

Table 5

Descriptive Statistics of Used Clues Based on Ames's (1966) Framework

\begin{tabular}{|c|c|c|c|c|c|c|c|}
\hline & Difficulty & Mean & $S D$ & & Difficulty & Mean & $S D$ \\
\hline \multirow[t]{3}{*}{ II } & easy & 4.76 & 9.18 & IX & Easy & 9.52 & 17.82 \\
\hline & difficult & 0.48 & 1.51 & & Difficult & 4.31 & 10.40 \\
\hline & total & 2.38 & 6.38 & & Total & 6.63 & 13.97 \\
\hline \multirow[t]{3}{*}{$\mathrm{V}$} & easy & 10.12 & 28.62 & XII & easy & 0.00 & 0.00 \\
\hline & difficult & 0.00 & 0.00 & & difficult & 12.86 & 27.68 \\
\hline & total & 4.50 & 19.08 & & Total & 7.14 & 21.19 \\
\hline \multirow[t]{3}{*}{ VII } & easy & 74.40 & 30.43 & & & & \\
\hline & difficult & 71.88 & 29.46 & & & & \\
\hline & total & 73.00 & 29.02 & & & & \\
\hline
\end{tabular}




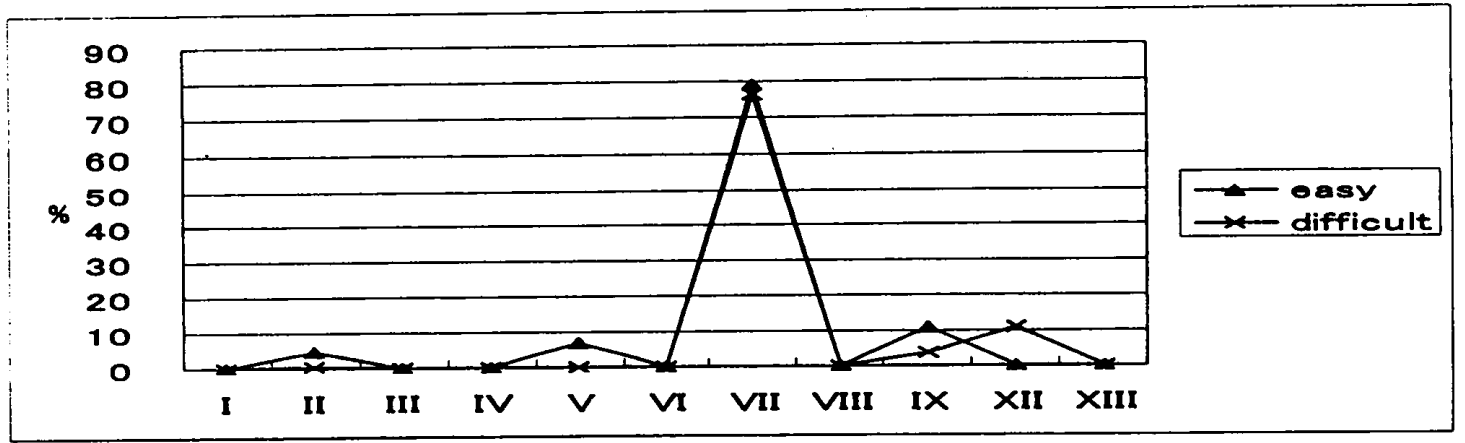

Figure 3. Ames's (1966) clues used by participants

That no interaction was found indicates that the kinds of contextual clues used were the same even though item difficulty changed, and VII was the dominant clue. Below is an example of participants using VII.

As I have no interest in this ceremony, the series of speeches seemed (_) to me. a. differential b. eternal c. managing d. restrictive

$<$ Participant \#21 (using VII: mood)>

"WELL, THE CHARACTER FEELS SOMETHING. THIS SENTENCE SAYS I HAVE NO INTEREST IN THIS CEREMONY, SO A WORD HAVING NEGATIVE MEANING WILL BE IN THE BLANK, SUCH AS BORING. THE CHARACTER DOES NOT HAVE A GOOD FEELING."

Tom needed ( ) for devoting himself to studying to pass the entrance examination of the high school.
a. chapel
b. entity
c. isolation d. refusal

$<$ Participant \#13 (using VII: setting)>

"WELL, IN ORDER TO PASS THE ENTRANCE EXAMINATION, LET ME SEE, SOMETHING IS NEEDED TO STUDY, SO SOMETHING WHICH AROUSES THE FEELING TO CONCENTRATE ON STUDYING, SUCH AS SOMETHING WHICH MAKES TOM FEELS LIKE STUDYING."

In most cases, the participants used either the setting or mood of the context to answer the questions, and this tendency was the same if the item difficulty varied. However, the frequency of using VII differed because in difficult items, some participants gave up answering because they were not sure of the meanings of some words in the contexts. For instance, Participant \#13 could not use contextual clues at all because she said she did not know the meaning of property in the following item: If you die without ( ), your property will go to the state. In the same item, Participant 
\#16 could not grasp the meaning of the latter half of the sentence, so he said it was very hard to imagine the word in the blank. Another example is from the item: Williams cleared his throat, made a few ( ) remarks welcoming everyone, then began his speech. Participant \#3 regarded the word in the brackets as an adjective, but since she persisted in trying to understand the meaning of remarks, which was an unknown word to her, she could not think of a suitable word. Her failure was also due to the misunderstanding of the phrase clear one's throat as gargling.

However, the participants did not always give up when they came across unknown words. In the following item: In the evening, a flock of birds flapped slowly across the ( ), Participant \#14 gave up on this item because she did not know the meaning of flock and flap. On the other hand, although Participant \#16 was also not sure of the meanings of these words, he utilized the minimum context of this sentence. That is, he sought the word coming after across the, not relying on the other part of the sentence, and his answer, road, was contextually appropriate. Therefore, the difference in the number of clues used between easy and different items mainly resulted from whether there were unknown words in the contexts or not, but in some cases, participants could overcome the difficulty.

In order to seek how often participants gave up answering when they had unknown words in the context, the protocol data was reexamined and it was found that two or more participants explicitly stated that they had unknown words in the contexts in four out of eight easy items and in eight out of 10 difficult items. What is interesting is that more than half of the participants (average: $74.84 \%$ ) who had unknown words in context reached some answers in most items of this kind (11 out of 12). This was the case when the test-takers could use the words near the blanks as clues. For example, let us consider the following items retrieved from easy items: Her charming ( ) has won her numerous admirers. Although three participants did not know the meaning of numerous admirers and one participant was not sure of the meaning of numerous, they produced answers such as face from the word, charming, which is located just before the blank. The same tendency could be applied to another easy item: Buddha was a social ( ) who condemned India's caste system. A total of five participants had some unknown words: one participant did not know Buddha, three did not know condemned, and the other participant could not understand the meanings of either condemned or caste. However, four out of five participants effectively used the clues and did not give up. For them, the words placed between the blank, social and who, were useful clues. This tendency was similar when they answered difficult items. For instance, in the item: Donna was swamped with congratulations from ( ) reporters when she won the prize, six participants could not understand swamped and one participant did not know the meaning of congratulations, but five out of seven participants produced answers such as many or numerous, because they thought the word had to modify reporters, located just after the brackets. 
On the contrary, in one item (Williams cleared his throat, made a few ( remarks welcoming everyone, then began his speech.), only one out of five participants who had unknown words could reach an answer. This would be because the unknown word to the other four participants was remarks, which is next to the brackets. Therefore, unknown words near brackets probably hinder identifying the word in the blank. This is indirectly supported by previous research (e.g., Aizawa, 1998; Carnine et al., 1984; Dubin \& Olshtain, 1993; Huckin \& Bloch, 1993) reporting that clues near unknown words were effective for correctly inferring the meanings of unknown words. From these results, it would be easier to ignore the words far from the blank to reach an answer, but if there is an unknown word near the brackets, it becomes a huge obstacle to identify the word in the blank.

\subsection{Choice Selection}

In this section, the other aspect, the choices, was focused upon from the perspective of test-taking processes. Firstly, it can be hypothesized that the use of elimination differed between easy and difficult items. Table 6 shows percentages of elimination used by all participants, whether or not they answered correctly, incorrectly, or produced no response. A chi-square test was conducted to see if there was a significant difference between easy and difficult items, and between correct and incorrect participants, but there was no significant difference at all $\left(\chi^{2}(1)=.008, p\right.$ $=.930$ ). In order to check whether the use of elimination had something to do with the proportions correct, a regression analysis and correlation analysis between the proportions using elimination and the proportions correct were conducted. The elimination proportions did not significantly predict the proportion correct $\left(\beta=-.45, r^{2}\right.$ $=.21, p=.059)$, but there was a significant negative moderate correlation between the use of elimination and proportion correct $(r=-.45, p=.029)$. This result matches the figures in Table 6, indicating more participants used elimination in difficult items.

Table 6

The Proportion Using Elimination

All participants Correct participants

Easy

Difficult
$55.95 \%$

$66.93 \%$ $59.38 \%$
$52.50 \%$
Incorrect participants

Why did the use of elimination relate with proportions correct? By examining the protocol data, it became apparent that there are two possible reasons: (a) the difficult items increased the proportions of elimination, and (b) elimination made the proportions correct lower. The former pattern was caused by difficult words or phrases in context and choices. Difficult items had more unknown words or uncertain words in the contexts for the participants, so they could not utilize the context fully, leading to a lack 
of clues to select one correct answer from four choices; therefore, they could not directly select one answer. This is a similar pattern to the examples in 3.1. As for the difficulty of words in choices, let us focus on the following item retrieved from difficult items.

Tom needed ( ) for devoting himself to studying to pass the entrance examination of the high school.

a. chapel. b. entity c. isolation* d. refusal

Nine participants could not select the correct answer, and among them, five explicitly said that they did not know the meaning of the word isolation, which was the answer. In such a case, participants gave up totally, or eliminated other choices first, leaving the choice, as with Participants \#4 and \#6 below.

$<$ Participant \#4>

"I CANNOT IMAGINE THE ANSWER WELL, BUT I DON'T THINK THE ANSWER IS chapel. I'M NOT SURE, BUT CHURCHES HAVE A chapel, SO I DON'T THINK THAT'S THE ANSWER. Refuse...refuse...IT HAS THE MEANING OF REJECTION, AND refuse OR refusal HAS A NEGATIVE MOOD. SO I ELIMINATE IT, TOO. I HAVEN'T SEEN THE WORDS IN CHOICE $b$, BUT I HAVE SEEN isolation, PERHAPS, THOUGH I CAN'T REMEMBER THE MEANING. THE ANSWER IS EITHER CHOICE $b$ OR $c$."

$<$ Participant \#6>

"WHAT'S THIS? CHOICE $d$ IS NOT THE ANSWER IF refusal HAS THE SAME MEANING AS refuse. I HAVE HEARD isolation, BUT I CAN'T REMEMBER IT. Chapel...I DON'T KNOW...chapel.... I WISH I KNEW THE MEANING OF devoting. CAN I ANSWER BY GUESSING? (OR YOU CAN REJECT SOME CHOICES.) WELL, CHOICE $d$ IS NOT THE ANSWER. (WHY IS THAT?) I THINK refusal IS THE NOUN FORM OF refuse, MEANING REJECTING. (THEN...) IT DOESN'T MATCH. WELL...IF chapel INDICATES THE PLACE, I THINK Tom NEEDS SOME PLACES. ANYWAY CHOICE $a$ IS FAIR. (YOU CAN PROCEED WITHOUT CONFIRMING THE ANSWER, BUT YOUR FINAL DECISION IS THAT IF THE CHOICE $a$ INDICATES SOME PLACES, THE ANSWER IS CHOICE $a$, RIGHT?) YES."

Both of them had heard the word isolation, but they did not remember its meaning. This made them leave the choice $c$, isolation, and start rejecting the possibility of other choices being correct answers. Hence, when there were unknown or unsure words either in context or in choices, which is often the case in difficult items, the use 
of elimination increased.

Another pattern is that elimination made the proportions correct lower. This pattern happened because in four out of 10 difficult items, three or more participants eliminated the correct choices, although this did not occur in easy items. Therefore, in order to seek the reasons of this, these items were examined one by one.

Lack of utilizing keywords

In ancient times, the ( ) from hunting may have been very gradual. a. answer b. change* c. show d. study

The first cause of eliminating the correct answer was lack of utilizing contexts. The word in the blank is the subject of this sentence, and gradual is a complement, and would be one of the most useful clues in this item. However, all of the five participants who eliminated the correct answer did not use gradual as a clue, or mistakenly used it. For example, one of the participants clung to the phrase, the () from hunting.

$<$ Participant \#5>

"answer from hunting, NO, SOMETHING FROM HUNTING...change, HUNTING show, change. I THINK IT'S change. Change from...NO, IT DOESN'T MAKE SENSE. Study from, study, answer from, ANSWER, hunting, ANSWER, study from...THE ANSWER IS STUDY, CHOICE $d$. (SO, YOU THINK WHETHER THE WORD MATCHES IN THIS PHRASE [the（） from hunting].) YES."

Two other participants eliminated change because they considered the time span. Participant \#9 regarded have been in the sentence as indicating that something was happening over a long period, and her intuition was that change happens only in the short term, so she deleted it. The other participants thought vice versa, as shown below.

$<$ Participant \#6>

"Show CAN BE AN ANSWER, AND study MATCHES THE CONTEXT IF IT MEANS METHOD, WELL... I THINK THE ANSWER IS CHOICE $d$. I THINK THE ANSWER IS CHOICE $b, c$, or $d$, RATHER THAN CHOICE $a$. CHOICE $b$ MATCHES THE CONTEXT IF CHANGE OCCURRED FROM THE ANCIENT TIMES TO NOW, BUT IF IT IS THE ANCIENT TIMES ONLY, IT IS NOT SUITABLE ENOUGH. SO I THINK THE ANSWER IS CHOICE $d$, study, INDICATING THE WAY OF HUNTING."

Therefore, because both of their views about change and the meaning of context did not match from the viewpoint of time span, they thought change did not make sense 
in the context. However, if they had utilized gradual in the context as a clue, their answers might have switched. It is also possible that they did not know the meaning of gradual, but they did not explicitly state this, so it remains unclear. Two other participants who eliminated change mistakenly recognized gradual as general, so they could not select the correct answer. In sum, all of the participants who eliminated the correct answer in this item could not make sufficient use of all the words in the context, especially gradual.

\section{Mood of vocabulary}

As I have no interest in this ceremony, the series of speeches seemed ( ) to me. a. differential $b$. eternal ${ }^{*}$ c. managing d. restrictive

The next example of eliminating correct answers was caused by the test-takers' intuitions toward the word. Seven participants excluded the correct answer, eternal, but most of them did not state the reason explicitly; they just deleted the word without saying why, or they just said that "IT JUST DOESN'T MATCH THE CONTEXT AT ALL (Participant \#7)." However, one participant clearly gave the reason as follows.

$<$ Participant \#6>

"WELL...differential IS SIMILAR TO different. To me MEANS FOR ME? THE WORD, eternal, REMINDS ME OF POSITIVE MOOD, AND THE WORD IN THE BLANK SHOULD BE THE ONE WITH A NEGATIVE MOOD."

This participant perceived a negative mood from the contexts, so she thought the words in the blank also had to be negative. The mood of the correct answer and the sentence did not suit in her mind so she rejected the possibility of choice $b$ being correct because the word, eternal, had a positive mood for her. Hence, the difference of mood could affect which choice test-takers select.

\section{Multiple meanings}

If you die without ( ), your property will go to the state.

a. densities b. fractions c. spheres d. successors*

In this item, the derivational form (succeed) of the correct answer (successor) has two totally different meanings (an antonym to fail and to be an heir), which led to a decrease in the proportion correct. Five out of six participants who eliminated the correct answer regarded the meaning of successor as a person who succeeds (as an antonym to fail), although it actually means heirs. Hence, they rejected successors since it did not match the context. The remaining participant (Participant \#17) took successors as meaning heirs, but still could not regard it as correct. She was the only one who 
could not select the correct answer among those who could correctly recognize the meaning of successors. Below is the protocol data produced by participant \#17.

$<$ Participant \#17>

"I KNOW THE MEANING OF CHOICE $d$, successor. IT'S AN HEIR. (DOES IT MAKE SENSE IN THE CONTEXT?) WELL, I AM PUZZLED. IF THE SITUATION OF THIS CONTEXT IS IN A COMPANY, THE WORD, successors, MIGHT BE SUITABLE. BUT IF THERE ARE DESCENDENTS, HEIRS ARE NOT USUALLY NEEDED. DOES THE WORD IN THE CHOICE $a$ MEAN WILL? I DON'T REMEMBER. THE ANSWER IS EITHER CHOICE $a$ OR $d$, BUT CHOICE $d$ DOES NOT FIT IN THE CONTEXT. BUT I DON'T REMEMBER WHETHER THE WORD IN CHOICE a MEANS WILL...WELL, success, if you die without successors...WELL, CHOICE $d$ DOES NOT MAKE SENSE. CHOICE $d$ INDICATES PEOPLE, AND WITHOUT PEOPLE, WITHOUT successors.... I DECIDE THE ANSWER. IT'S CHOICE $a$, BELIEVING IT MEANS WILL."

From the protocol, it can be inferred that her concepts of successors is narrower than it actually is. She thought successors were those who replaced others in company positions, not those who received money or land after someone's death. Because of this, she could not reach the correct answer. However, her case is an exception, and most of the participants who excluded choice $d$ thought successors were those who succeeded, as antonym to those who fail.

\section{Multiple senses}

All the cars are inspected for the ( ) before they leave the factory.

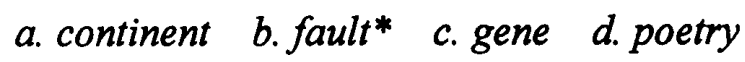

This item has a similar result to the last item discussed in regard to multiple meanings. Eight participants rejected the correct choice, fault, and their common reason was that they knew the meaning of fault but they could not apply it in the context. In other words, they adhered to only one sense of the words and lacked flexibility of the senses. Most of them regarded the meaning of fault as either shippai (failure) or machigai (mistake), and they could not link it with kekkan (defect), which would be more suitable to the context above. This becomes clearer when comparing those who answered correctly and incorrectly (underlines are added by the author to see the comparisons more clearly).

$<$ Participant \#3: incorrect>

"Fault MEANS SOMETHING RELATED TO FAILURE <SHIPPAI>, SO IT HAS NOTHING TO DO WITH THE CONTEXT. FOR FAILURE $<$ SHIPPAI $>$ ?" 
$<$ Participant \#7: incorrect>

"Fault MEANS FAILURE <SHIPPAI>. FOR THE FAILURE<SHIPPAI> ALL THE CAR IS..., IT SEEMS NOT TO MAKE SENSE."

$<$ Participant \#9: correct>

"Fault MEANS MISTAKE <MACHIGAI> OR SOMETHING LIKE THAT, SO IF THE WORD IN THE BLANK IS fault, IT MEANS THE CARS ARE INSPECTED FOR SOME MISTAKE <MACHIGATTA-TOKORO> OR WRONG PARTS <WARUI-TOKORO $>$ BEFORE THEY ARE BROUGHT TO THE FACTORY. IT MAKES SENSE, SO THE ANSWER IS CHOICE $b . "$

$<$ Participant \#18: correct >

"Fault HAS A MEANING OF FAILURE <SHIPPAI $>$ OR DEFECT <KETTEN>, SO THEY REPAIR THE WRONG PARTS <WARUI-BUBUN $>$ :"

By contrasting these two types of test-takers, it is apparent that those who answered incorrectly repeatedly used the same Japanese translation, shippai, and would not modify it to make it fit the context. On the other hand, those who succeeded in reaching the correct answer paraphrased the meaning of fault two or three times, as wrong parts (warui-tokoro or warui-bubun) or defect (ketten). Therefore, the items whose correct answer had multiple senses were difficult when test-takers could not grasp the meanings flexibly. When the target words had either multiple meanings or multiple senses, the items became more difficult since it was possible that the test-takers mistakenly eliminated the correct answer without noticing the other meaning or sense. This is a different tendency from Frazier and Rayner (1990), whose result suggested that a sentence including words with multiple meanings and with multiple senses bore different results. However, their study measured L1 participants' reading time while this study focused on L2 students' test-taking processes, so it is reasonable for the tendency to be different.

In summary, test-takers rejected the correct answers when they could not utilize the context fully, when they regarded the mood of correct answers as different from that of context, and when the correct answers had multiple meanings or senses. Most of errors resulted from lack of vocabulary knowledge, especially quality or depth of word knowledge. The type of vocabulary tests in context used in this study would be made to assess breadth of vocabulary knowledge, rather than depth, since they only seem to measure whether test-takers know vocabulary meaning. However, the examination of the protocol data indicates that the test sometimes requires knowledge beyond word meanings, which partially supports Morimoto's (2006b) results that this type of test had a stronger partial correlation with reading ability or collocation knowledge than 
knowledge of vocabulary meaning. Integrating quantitative and qualitative research about contextualized vocabulary tests would clarify what this kind of vocabulary test actually measures.

\section{Conclusion}

Firstly, the research questions were examined. The answer to RQ1 (Is there any difference of use of context clues between easy and difficult items of vocabulary tests in context?) is that there is some difference in both the kinds of clues and the number of clues. Test-takers used a greater variety of clues in easy items, such as clues of world knowledge, and also used more clues in easy items than in difficult items. As for RQ2 (Is there any difference in the processes of choosing an answer in multiple-choice vocabulary tests in context between easy and difficult items?), the participants used elimination more in difficult items. This sometimes lead to elimination of the correct answer because of the following reasons: lack of utilizing keywords, different mood of vocabulary from that of context, and the word in the correct answer has multiple meanings or senses.

In terms of limitations of this study, the number of participants and test items were not large because collecting thinking-aloud data requires much time for conducting research as well as writing down what the participants say. Using many items will offer more variety in test-taking processes, so it would help in investigating why participants cannot reach correct answers or the construct of vocabulary tests in context. Moreover, although thinking-aloud is good way to investigate the test-taking process, it might impair students' ability (Cordón \& Day, 1996); hence, the results should be interpreted with caution. Also, this study restricted the length of context within a sentence for each item, so using a longer context could generate more diverse processes.

Lastly, the pedagogical implications of this study are that the most dominantly used clues are located in the same sentence as the target word, so utilizing the clue words with world knowledge will increase the probability of reaching correct answers in vocabulary tests in context. By learning how to use the clues, students will be able to exploit their vocabulary knowledge in taking tests. Furthermore, teachers should be cautious in using elimination strategies since this sometimes leads to rejecting the correct answer. In order to avoid this problem, it is necessary to teach vocabulary knowledge from various aspects such as plural meanings or mood. Teaching word meaning by matching with their Japanese translations is a good first step to acquiring word meaning, but we have to be careful to compensate for its deficiencies later.

\section{Acknowledgement}

I am grateful to Professor Yuji Ushiro, Ms. Yuko Hijikata, and Ms. Chikako Nakagawa for their valuable comments. 
Note

1) $\eta^{2}=$ eta squared (effect size) $0.010 \leqq$ eta squared (small) $\leqq 0.059 ; 0.059 \leqq$ eta squared (medium) $\leqq 0.138 ; 0.138 \leqq$ eta squared (large; Cohen, 1988, pp. 284-287). Effect size of 0.009 and below is regarded as having no effect.

2) The words in the brackets were those of the researcher. The protocols produced in Japanese were written in uppercase letters, following Nikolov (2006).

\section{References}

Aizawa, K. (1998). Lexical inferencing cues in reading and Japanese learners of English. JACET Bulletin, 29, 1-19.

Ames, W. S. (1966). The development of a classification scheme of contextual aids. Reading Research Quarterly, 2, 57-82.

Beck, I. L., McKeown, M. G., \& McCaslin, E. S. (1983). Vocabulary development: All contexts are not created equal. The Elementary School Journal, 83, 177-181.

Bengeleil, N. F., \& Paribakht, T. S. (2004). L2 reading proficiency and lexical inferencing by university EFL learners. Canadian Modern Language Review, 61, 225-249.

Carnine, D., Kameenui, E., and Coyle, G. (1984). Utilization of contextual information in determining the meaning of unfamiliar words. Reading Research Quarterly, 19, 188-203.

Cohen, J. (1988). Statistical power analysis for the behavioral sciences (2nd ed.). Hillsdale, NJ: Erlbaum.

Cordón, L. A., \& Day, J. D. (1996). Strategy used on standardized reading comprehension tests. Journal of Educational Psychology, 88, 288-295.

Dubin, F., \& Olshtain, E. (1993). Predicting word meanings from contextual clues: evidence from L1 readers. In T., Huckin, M. Haynes, \& J. Coady (Eds.), Second Language Reading and Vocabulary Learning (pp.181-202).

Frazier, L., \& Rayner, K. (1990). Taking on semantic commitments: Processing multiple meanings vs. multiple senses. Journal of Memory and Language, 29, 181-200.

Greidanus, T., \& Nienhuis, L. (2001). Testing the quality of word knowledge in a second language by means of word associations: Types of distractors and types of associations. Modern Language Journal, 85, 467-477.

Haastrup, K. (1991). Lexical inferencing procedures or talking about words. Gunter Narr Verlag Tubingen: Tubingen.

Henning, G. (1991). A study of the effects of contextualization and familiarization on responses to the TOEFL vocabulary test items. TOEFL Research Report RR-35, Educational Testing Services: Princeton.

Huckin, T. \& Bloch, J. (1993). Strategies for inferring word-meaning in context: a cognitive model. In T. Huckin, M. Haynes and J. Coady (Eds.), Second language reading and vocabulary learning (pp.153-178). Greenwood.

Hughes, A. (2003). Testing for language teachers. Cambridge University Press.

Juel, C. (1980). Comparison of word identification strategies with varying context, word type, and reader skill. Reading Research Quarterly, 15, 358-376.

Laufer, B., Elder, C., Hill, K., \& Congdon, P. (2004). Size and strength: Do we need 
both to measure vocabulary knowledge? Language Testing, 21, 202-226.

Marshalek, B. (1981). Trait and process aspects of vocabulary knowledge and verbal ability (Tech. Rep. No. 15). CA: Stanford University, School of Education.

McCullough, C. M. (1958). Context aids in reading. Reading Teacher, 11, 225-229.

Mondria, J.-A., Boer, M. W.-T. (1991). The effects of contextual richness on the guessability and the retention of words in a foreign language. Applied Linguistics, 12, 249-267.

Mori, Y. (1999). Beliefs about language learning and their relationship to the ability to integrate information from word parts and context in interpreting novel kanji words. The Modern Language Journal, 83, 534-547.

Mori, Y. (2002). Individual differences in the integration of information from context and word parts in interpreting unknown kanji words. Applied Psycholinguistics, $23,375-397$.

Mori, Y. (2003). The roles of context and word morphology in learning new kanji words. The Modern Language Journal, 87, 404-420.

Morimoto, Y. (2006a). Goi test no keisiki ga goi chishiki to dokkai nouryoku no sokutei ni oyobosu eikyo [The effect of vocabulary test types on measuring word knowledge and reading ability]. STEP Bulletin, 18, 77-91.

Morimoto, Y. (2006b). Comparison between matching- and supplying-format in multiple choice vocabulary tests. JLTA Journal, 9, 73-85.

Nikolov, M. (2006). Test-taking strategies of 12- and 13-year-old Hungarian learners of EFL: Why whales have migraines. Language Learning, 56, 1-51.

Pearson, P. D., \& Studt, A. (1975). Effects of word frequency and contextual richness on children's word identification abilities. Journal of Educational Psychology, 67, 89-95.

Perkins, K., \& Linnville, S. E. (1987). A construct definition study of a standardized ESL vocabulary test. Language Testing, 4, 125-141.

Pike, L. W. (1979). An evaluation of alternative item formats for testing English as a foreign language. TOEFL Research Report RR-79-6, Educational Testing Services: Princeton.

Read, J. (2000). Assessing vocabulary. Cambridge University Press.

Schedl, M., Thomas, N., \& Way, W. (1995). An investigation of proposed revisions to section 3 of the TOEFL test. TOEFL Research Report RR-94-42, Educational Testing Services: Princeton.

Sternberg, R. J. \& Powell, J. S. (1983). Comprehending verbal comprehension. American Psychologist, 38, 878-893.

Takayama, Y. (2003). Inference of unknown words in reading comprehension among Japanese EFL high school students. KATE (Kanto-koshinetsu Association of Teachers of English) Bulletin, 17, 89-99. 